This item was submitted to Loughborough's Research Repository by the author.

Items in Figshare are protected by copyright, with all rights reserved, unless otherwise indicated.

\title{
A novel two-way method for dynamically coupling a hydrodynamic model with a discrete element model (DEM)
}

PLEASE CITE THE PUBLISHED VERSION

https://doi.org/10.1007/s42241-018-0081-y

\section{PUBLISHER}

Springer (C) China Ship Scientific Research Center

\section{VERSION}

AM (Accepted Manuscript)

\section{PUBLISHER STATEMENT}

This is a post-peer-review, pre-copyedit version of an article published in Journal of Hydrodynamics. The final authenticated version is available online at: https://doi.org/10.1007/s42241-018-0081-y

\section{LICENCE}

CC BY-NC-ND 4.0

\section{REPOSITORY RECORD}

Xiong, Yan, Qiuhua Liang, Samantha Mahaffey, Mohamed Rouainia, and Gang Wang. 2019. "A Novel Twoway Method for Dynamically Coupling a Hydrodynamic Model with a Discrete Element Model (DEM)”. figshare. https://hdl.handle.net/2134/36564. 


\title{
A novel two-way method for dynamically coupling a hydrodynamic model with a Discrete Element Model (DEM)*
}

Yan Xiong ${ }^{1}$; Qiuhua Liang ${ }^{1,2-*}$; Samantha Mahaffey ${ }^{3}$; Mohamed Rouainia ${ }^{3}$; Gang Wang ${ }^{1}$;

${ }^{1}$ State Key Laboratory of Hydrology-Water Resources and Hydraulic Engineering, Hohai University, Nanjing, 210098, China

${ }^{2}$ School of Architecture, Building and Civil Engineering, Loughborough University, UK

${ }^{3}$ School of Engineering, Newcastle University, Newcastle upon Tyne, England, UK

\begin{abstract}
The effect of floating objects has so far been little considered for hazard risk assessment and structure design, despite being an important factor causing structural damage in flood-prone and coastal areas. In this work, a novel twoway method is proposed to fully couple a shock-capturing hydrodynamic model with a Discrete Element Model (DEM) for simulation of complex debris-enriched flow hydrodynamics. After being validated against an idealized analytical test, the new coupled model is used to reproduce flume experiments of floating debris driven by dam-break waves. The numerical results agree satisfactorily with the experimental measurements, demonstrating the model's capability and efficiency in simulating the complex fluid-debris interactions induced by violent shallow flows.
\end{abstract}

Key words: Coupled model; Discrete element model; Extreme hydraulic conditions; Floating debris; Shallow Water Equations; Godunov-type finite volume method

\footnotetext{
* This work is supported by the China Scholarships Council (No. 201606710054), the UK NERC SINATRA and TENDERLY projects (grant no. NE/K008781/1), and the State Major Project of Water Pollution Control and Management (2017ZX07603-001).
}

Biography: Yan Xiong (1992-), Female, Ph. D. Candidate, E-mail: xiongyan@hhu.edu.cn

*Corresponding author: Qiuhua Liang, E-mail: Q.Liang@lboro.ac.uk 
In recent years, extreme water-related natural hazards, e.g. flash floods, tsunamis and storm surges, have become more frequent and attracted increasing attention from the public, scientists and other relevant communities. One of the striking features of these water-related events is that dense floating debris, of all shapes and sizes, may be carried along by the highly transient water flows. The floating debris may significantly worsen the devastation of these disasters by impacting and blocking structures, and subsequently causing further damage [1]. Many field investigations imply that water flow alone is much less destructive than debris-enriched flow during a hazard event [2]. However, the role of floating objects has not been adequately considered in the risk assessment frameworks and the design codes in flood-prone and coastal areas although new research in the topic has become increasingly reported in recent years ${ }^{[3-5]}$.

Numerical modelling has become an indispensable tool for the simulation of the interactive dynamics between fluid flows and floating objects. This is usually achieved by coupling two different types of models using one of the three types of coupling methods: one-way solid-tofluid coupling, one-way fluid-to-solid coupling, and two-way dynamic coupling [6]. Both types of one-way coupled models do not consider the feedback of the recipient phase to the driving phase, and are only applicable to certain simple cases where the dynamics of either the fluid or the solid phase is less predominant than the interaction between them ${ }^{[7]}$.

Two-way dynamic coupling methods take into account the dynamic interaction between the fluid flow and solids; whilst the fluid flow moves the solids, the motion of the solids imposes counter forces/effects on the fluid and changes its dynamics. Researchers have attempted to develop two-way coupled models for fluidsolid interaction using hydraulic models and force analysis methods ${ }^{[8]}$. A force analysis method considers buoyant force and drag force as the two most representative forces determining the interaction between fluid and solid ${ }^{[9]}$. The drag force is normally estimated using empirical formulas containing a drag coefficient, $C_{D}$. Expressions/values of $C_{D}$ are normally provided empirically according to the shape of the object under consideration and flow conditions [10]. Although certain models adopting this approach have been reported to be successful in simulating specific types of floating objects, e.g. ice floes and logs [11-13], the floating objects observed during a tsunami or extreme flood event are much more complex and may come in different shapes and sizes. Furthermore, the value of the drag coefficient for calculating drag force is normally estimated using empirical formula or values suggested by physical experiments under idealized flow conditions. There is no reliable approach to accurately quantify drag coefficient for the simulation of various floating debris in highly transient flow conditions during an extreme flood event. Therefore, there is a clear research gap and need in the development of computationally more efficient models for accurate simulation of the complex fluid-debris dynamics driven by large-scale violent shallow water flows.

This letter aims to briefly introduce our innovative but preliminary work of developing a novel two-way coupled model consisting of a shock-capturing hydrodynamic model solving the shallow water equations (SWEs) and a discrete element model (DEM) for describing the movement of floating debris. In order to avoid using parameterized drag force to foster the coupling, the fluid-solid interaction is encapsulated through hydrostatic and hydrodynamic pressure forces, which is consistent with the theory of shallow water flow. This new two-way dynamic coupling approach effectively avoids introducing uncertain parameters (e.g. drag coefficient) for force calculation. The resulting model is applicable to the more complex environmental flows with highly transient flow conditions and is validated against analytical and experimental test cases with satisfactory results obtained.

For shallow flows with dominated dynamics in one spatial dimension, the 1D SWEs may be written in a matrix form as

$$
\frac{\partial \mathbf{q}}{\partial t}+\frac{\partial \mathbf{f}}{\partial x}=\mathbf{s}
$$

where $t$ and $x$ represent respectively the time and horizontal coordinate, and $\mathbf{q}, \mathbf{f}$ and $\mathbf{s}$ are the vectors containing the flow variables, fluxes and source terms. The vector terms are provided as follows

$$
\begin{aligned}
& \mathbf{q}=\left[\begin{array}{ll}
\eta & u h
\end{array}\right]^{\mathrm{T}} ; \mathbf{f}=\left[\begin{array}{ll}
u h & u^{2} h+g\left(\eta^{2}-2 \eta z_{b}\right) / 2
\end{array}\right]^{\mathrm{T}} ; \\
& \mathbf{s}=\left[\begin{array}{ll}
0 & -\tau_{b x} / \rho-g \eta \partial z_{b} / \partial x+S_{v x}-S_{p x}
\end{array}\right]^{\mathrm{T}}
\end{aligned}
$$

where $\eta$ and $z_{b}$ represent the water surface elevation and bed elevation above datum with $h=\eta-z_{b}$ being the total water depth; $u$ is the depth-averaged velocity; $g$ is the acceleration due to gravity; $\rho$ denotes the water density; $-\partial z_{b} / \partial x$ defines the bed slope in the $x$-direction; $\tau_{b x}$ is the bed friction stress; $S_{v x}$ is the turbulent (viscous) term; and $S_{p x}$ contains the extra forces (stresses) to facilitate the coupling with a DEM model, which will be introduced in more detail later. Herein, the governing SWEs are solved using a second-order finite volume Godunov-type scheme implemented with an HLL approximate Riemann solver for evaluating interface fluxes. The second-order accuracy in both space and time is achieved through a two-step MUSCL-Hancock method. Detailed implementation of the numerical method can be found in Liang and Borthwick ${ }^{[14]}$. 
For the adopted DEM model, the following discretized equations are normally used to calculate the translational and rotational motions of the elements:

$m_{i} \frac{d \mathbf{w}_{i}}{d t}=\mathbf{F}_{i}^{p}+\mathbf{F}_{i}^{f}+\mathbf{F}_{i}^{g} ; I_{i} \frac{d \mathbf{\omega}_{i}}{d t}=\mathbf{T}_{i}^{p}+\mathbf{T}_{i}^{f}$

where $i$ is the index of the elements/particles; $m_{i}$ and $I_{i}$ are the mass and moment of element $i ; \mathbf{w}_{i}$ and $\boldsymbol{\omega}_{i}$ are the velocity and angular velocity; $\mathbf{F}_{i}^{p}$ is the sum of contact forces acting on element $i$ by other elements or fixed structures; $\mathbf{F}_{i}^{g}$ represents the vertical gravity force; and $\mathbf{F}_{i}^{f}$ is the forces acting on the element $i$ by the surrounding fluid; $\mathbf{T}_{i}^{p}$ and $\mathbf{T}_{i}^{f}$ are torques acting on the centroid of element $i$, respectively generated by $\mathbf{F}_{i}^{f}$ and $\mathbf{F}_{i}^{g}$. For a particle/object under consideration, the combined forces and torques are used to calculate the acceleration, velocity and displacement at every time step. In this study, circles or spheres are adopted to idealize the floating objects.

The fluid forces are consisted of buoyancy, $\mathbf{F}_{i}^{b}$, and the sum of hydrostatic and hydrodynamic forces, $\mathbf{F}_{i}^{h}$. The joint forces (vectors) are used to couple the SWE model with the DEM model, i.e.

$\mathbf{F}_{i}^{f}=\mathbf{F}_{i}^{b}+\mathbf{F}_{i}^{h}$

In the vertical direction, the buoyancy is calculated as $\left|\mathbf{F}_{i}^{b}\right|=F_{i}^{b}=\rho g V_{i}$

where $V_{i}$ is the volume of fluid displaced by the particle under consideration, i.e. element $i$. The buoyancy will be also compared with the gravity to determine the vertical position of element $i$ relative to the fluid surface, e.g. "completely submerged" or "floated". This will accordingly allow the calculation of the submerged area for fluid impact calculation.

In the horizontal direction, hydrostatic and hydrodynamic forces are calculated from the flow variables predicted by the SWE model. Firstly, the total pressure $p_{i}^{h}$ at an arbitrary point on particle $i$ is given by

$p_{i}^{h}=p_{i}^{s}+p_{i}^{d}=\rho g z+\rho\left(\beta u-w_{i}\right)^{2}$

where $p_{i}^{s}$ and $p_{i}^{d}$ are respectively the static and dynamic components of point pressure; $z$ is the water depth from the surface to the point of interest; $u$ and $w_{i}$ are the depth-averaged flow velocity and the velocity of particle $i$, predicted respectively by the SWE model and $\mathrm{DEM} ; \beta$ is a correction factor introduced to correct the surface flow velocity and $\beta=1.1$ is used in this work for flows in natural streams and floods conditions ${ }^{[15]}$.
The total fluid impact that moves the debris/particle can be then obtained as follows

$\left|\mathbf{F}_{i}^{h}\right|=F_{i}^{h}=p_{i B}^{h} S_{B}-p_{i F}^{h} S_{F}$

where the subscripts, $B$ and $F$, indicate the 'Back' and 'Front' of the particle, and $S_{B}$ and $S_{F}$ are the areas of the 'Back' side and 'Front' side of the particle that are submerged in the water, which is calculated after the vertical position of the particle is determined (through the relationship between $\mathbf{F}_{i}^{b}$ and gravity).

When coupling the SWE model with the DEM, only the horizontal forces are necessary to be considered and the joint counter-force/stress added to the source terms in Eq. (2) is given by

$S_{p x}=p_{i}^{h} / \rho$

The new two-way coupled model is validated against an analytical test, and then applied to reproduce a series of dam-break flume experiments with floating objects. The idealized case of an initially stagnant solid ball moving by a slow steady flow is first considered to test the new fluid-solid coupled model. The ball is assumed to float in the water from the start of the simulation, with the buoyancy equal to gravity. For slow steady flows, the water levels at the front and back of the ball may be assumed to be identical, leading to $S_{B}=S_{F}$. Therefore, the static component of the fluid pressure force is 0 . The total fluid impact on the ball is therefore equal to the dynamic component of the pressure force. Subsequently the velocity of the ball is analytically derived as:

$w_{i}(t)=\beta u-\frac{\beta u m_{i}}{\beta u S_{F} \rho t+m_{i}}$

Assuming the initial location of the ball is $s_{0}$, the displacement, $s_{i}$, and acceleration, $a_{i}$, can be also analytically derived and given as follows:

$s_{i}(t)=\beta u t-\frac{m_{i}}{S_{F} \rho} \ln \left(\frac{S_{F} \rho \beta u}{m_{i}} t+1\right)+s_{0}$

$a_{i}(t)=\frac{\beta^{2} u^{2} \rho S_{F} m_{i}}{\left(\beta u S_{F} \rho t+m_{i}\right)^{2}}$

In the simulation, the steady flow is assumed to have a constant water depth of $0.4 \mathrm{~m}$ and velocity of $0.5 \mathrm{~m} / \mathrm{s}$ in a $20 \mathrm{~m}$ flume. A small ball of radius $0.02 \mathrm{~m}$ and density $500 \mathrm{~kg} / \mathrm{m}^{3}$ is released instantly at $t=0$ at a location $0.5 \mathrm{~m}$ from the left boundary (i.e. $s_{0}=0.5 \mathrm{~m}$ ). The numerical results in terms of acceleration, velocity and $x$-position of the ball are presented in Fig. 1 and compared with the analytical solutions. The numerical predictions agree perfectly well with analytical solutions, confirming that the new two-way coupling method can effectively simulate the motion of floating solids driven by simple steady flows. 
(a)

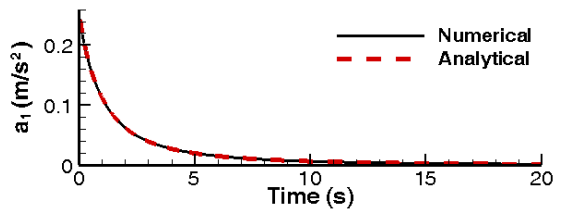

(b)

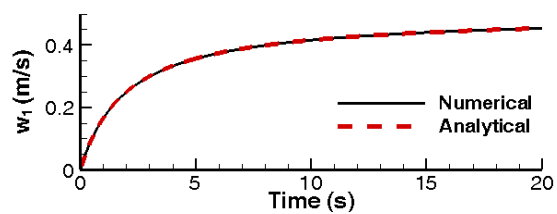

(c)

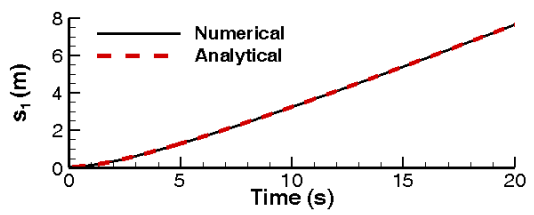

Fig. 1 Comparison of numerical and analytical solutions for a solid ball moving in a steady flow: (a) acceleration; (b) velocity; (c) $x$-position.

A series of flume experiments have been designed and carried out to investigate the interaction between floating debris (wooden sticks) and flows at the hydraulic laboratory in Hohai University, China. The flume is $35.5 \mathrm{~m}$ long, $1 \mathrm{~m}$ wide and $1.3 \mathrm{~m}$ deep. The extreme flow conditions were created by instantly opening a gate installed at the upstream section to generate dam-break waves. Calibrated wave gauges were installed along the channel near the wall to record time histories of water depth/level. Details of the experiment set up can be found in Liang et al. ${ }^{[16]}$. A camera mounted at the top of the flume was used to capture the flow dynamics and the motions of woody debris. Four different sizes of cylindrical woody debris were used in the experiments. One piece of the debris was initially placed at $0.75 \mathrm{~m}$ downstream of the gate in each test. Herein, three experimental tests with different debris and different water depths upstream and downstream of the dam are primarily selected and reproduced by the current coupled SWE-DEM model.

The predicted and measured time series of water depth and positions of the floating objects are compared in Fig. 2. For the time series of water depth, the black dashed lines and red solid lines respectively represent the experimental measurements and numerical predictions. For the debris positions, the measured data (extracted from the videos) and numerical results are denoted respectively by black circles and red solid lines. Both of the water depths and $x$-positions of the debris are satisfactorily simulated and compared reasonably well with the measurements. Particularly, the arrival times and the peak water levels of the dam break waves are all well predicted. The moments when the wooden sticks are activated are also correctly captured in all tests. The slight overestimation of the water level at final equilibrium state may be caused by the limitations of the over-simplified 1D SWE model. But the movement of the floating objects is dominated by advancing wave front and less relevant to the final state of the flow. The predicted trajectories of floating objects do not seem to be affected by the less accurate prediction of the final steady background flow. Successful reproduction of these experimental tests demonstrates the model's capability in simulating the complex behaviours of floating debris driven by violent dam-break flows. This confirms that the proposed two-way coupling approach is effective to capture the highly transient interactions between floating debris and fluid flows.

(a)
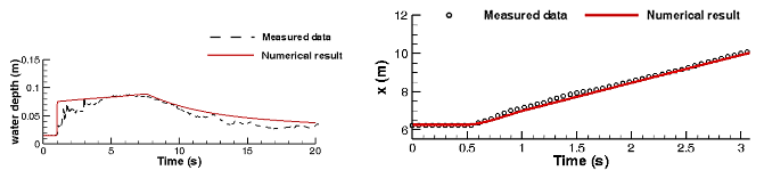

(b)
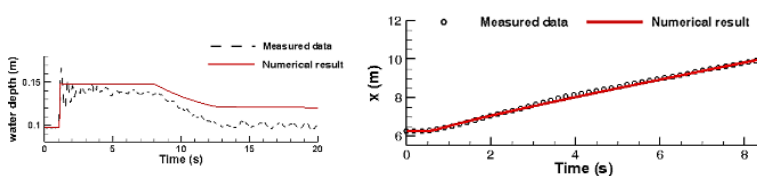

(c)
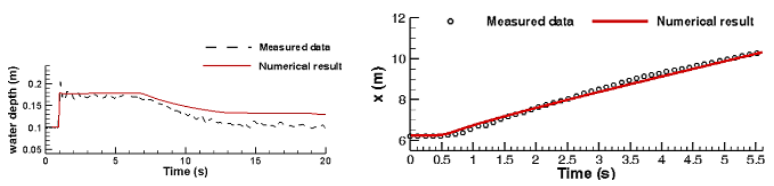

Fig. 2 Predicted and measures time serious of water depth and $x$-position of floating object of (a) Case 1 (upstream: $0.2 \mathrm{~m}$; downstream: $0 \mathrm{~m}$ ); (b) Case 3 (upstream: $0.2 \mathrm{~m}$; downstream: $0.1 \mathrm{~m}$ ); (c) Case 4 (upstream: $0.3 \mathrm{~m}$; downstream: $0.1 \mathrm{~m}$ ).

In this work, a new two-way coupling approach is proposed to dynamically couple a 1D SWE model with a DEM to simulate the complex behaviours of floating debris driven by highly transient shallow flows. Within the proposed modelling framework, a 1D shockcapturing SWE numerical model is fully coupled to a DEM model through buoyancy, hydrostatic and hydrodynamic forces. The resulting coupled model is then validated against analytical and experimental test cases with satisfactory results obtained. This preliminarily demonstrates the validity of the coupling approach and capability of the resulting model to accurately capture the highly complex dynamics of the floating objects driven by highly transient shallow flows. More systematic model validation is currently being undertaken and will be reported in the forthcoming full paper. Although it is currently presented in $1 \mathrm{D}$, the model can be readily extended to $2 \mathrm{D}$ for wider applications, especially for large-scale simulation of floating debris driven by flash floods, storm surges or tsunamis. This will be achieved in future work.

\section{Reference}


[1] A. Hatzikyriakou, N. Lin, J. Gong, S. Xian, X. Hu, and A. Kennedy, "Component-based vulnerability analysis for residential structures subjected to storm surge impact from Hurricane Sandy," Natural Hazards Review, vol. 17, p. 05015005, 2015.

[2] T. Rossetto, N. Peiris, A. Pomonis, S. Wilkinson, D. Del Re, R. Koo, et al., "The Indian Ocean tsunami of December 26, 2004: observations in Sri Lanka and Thailand," Natural Hazards, vol. 42, pp. 105-124, 2007.

[3] I. Nistor, N. Goseberg, J. Stolle, T. Mikami, T. Shibayama, R. Nakamura, et al., "Experimental Investigations of Debris Dynamics over a Horizontal Plane," Journal of Waterway, Port, Coastal, and Ocean Engineering, vol. 143, p. 04016022, 2016.

[4] I. Nistor, N. Goseberg, and J. Stolle, "Tsunami-Driven Debris Motion and Loads: A Critical Review," Frontiers in Built Environment, vol. 3, p. 2, 2017.

[5] A. Ardianti, H. Mutsuda, K. Kawawaki, and Y. Doi, "Fluid structure interactions between floating debris and tsunami shelter with elastic mooring caused by runup tsunami," Coastal Engineering, vol. 137, pp. 120$132,2018$.

[6] J. F. O'Brien, V. B. Zordan, and J. K. Hodgins, "Combining active and passive simulations for secondary motion," IEEE Computer Graphics and Applications, vol. 20, pp. 86-96, 2000.

[7] T.-R. Wu, C.-R. Chu, C.-J. Huang, C.-Y. Wang, S.-Y. Chien, and M.-Z. Chen, "A two-way coupled simulation of moving solids in free-surface flows," Computers \& Fluids, vol. 100, pp. 347-355, 2014.

[8] L.-c. Qiu, F. Jin, P.-z. Lin, Y. Liu, and Y. Han, "Numerical simulation of submarine landslide tsunamis using particle based methods," Journal of Hydrodynamics, vol. 29, pp. 542-551, August 012017.

[9] J. Zhao and T. Shan, "Coupled CFD-DEM simulation of fluid-particle interaction in geomechanics," Powder technology, vol. 239, pp. 248-258, 2013.

[10] W. Zhong, A. Yu, X. Liu, Z. Tong, and H. Zhang, "DEM/CFD-DEM modelling of non-spherical particulate systems: theoretical developments and applications," Powder Technology, vol. 302, pp. 108152, 2016.

[11] V. Ruiz-Villanueva, E. B. Castellet, A. Díez-Herrero, J. M. Bodoque, and M. Sánchez-Juny, "Two dimensional modelling of large wood transport during flash floods," Earth Surface Processes \& Landforms, vol. 39, pp. 438-449, 2014.

[12] M. A. Hopkins, "Discrete element modeling with dilated particles," Engineering Computations, vol. 21, pp. 422-430, 2004.

[13] L. Chen, L. Sun, J. Zang, A. J. Hillis, and A. R. Plummer, "Numerical study of roll motion of a 2-D floating structure in viscous flow," Journal of Hydrodynamics, vol. 28, pp. 544-563, August 012016.

[14] Q. Liang and A. G. Borthwick, "Adaptive quadtree simulation of shallow flows with wet-dry fronts over complex topography," Computers \& Fluids, vol. 38, pp. 221-234, 2009.

[15] V. T. Chow, "Open channel hydraulics," 1959.
[16] Q. Liang, K.-c. Chen, H. Jingming, Y. Xiong, W. Gang, and J. Qiang, "Hydrodynamic modelling of flow impact on structures under extreme flow conditions," Journal of Hydrodynamics, Ser. B, vol. 28, pp. 267-274, 2016. 